\title{
EDITORIAL
}

\section{New horizons for cancer gene therapy}

\author{
Cancer Gene Therapy (2014) 21, 1; doi:10.1038/cgt.2013.80
}

Despite recent advances in traditional chemotherapy, radiation therapy and surgery, cancer remains a leading cause of morbidity and mortality. ${ }^{1}$ Although this in part illustrates the challenges in developing effective therapies, it is also indicative of the limitations of traditional approaches. The development of new therapies must balance the goal of enhanced efficacy against the reality of off-target toxicity that so often prevents the effective application of cytotoxic regimens. Cancer gene therapy holds the promise of allowing for more targeted delivery of therapeutics as well as the potential to target specific pathways.

Advances in sequencing technology over the last 10 years have resulted in unprecedented discoveries of genes and pathways involved in tumorigenesis revealing potential targets for gene and cellular therapies. With this data in hand, novel approaches such as siRNA delivery to block a critical pro-growth pathway or delivery of a gene coding for a pro-apoptotic inducer can be realized. ${ }^{2,3}$ Such a tailored approach has the potential to improve efficacy and minimize toxicity.

Immunotherapy is garnering increasing attention as a viable strategy for the treatment of cancers beyond melanoma and renal cell carcinoma. As an increased understanding of the mechanisms of immune regulation has evolved, novel targets and strategies to manipulate those mechanisms have proven effective in the clinic. Gene modified T-cell therapy has shown promise in the clinic for a variety of tumor histologies. ${ }^{4}$

New vectors allowing for targeted gene delivery, enhanced gene expression and evasion of clearance have been developed and are being validated for translation to the clinic. These new classes of vectors are based on nanomaterials, hybrid bacterial viruses and novel engineering of cell-based carriers. Tissue-specific targeting holds promise for achieving that elusive goal of eliminating off-target toxicity and reducing the morbidity of systemic cancer treatment. ${ }^{5}$

These advances have also resulted in the application of gene therapy as an adjuvant to traditional chemotherapy, radiation therapy and surgery. Gene therapy can be used in this regard to allow for lower doses of standard regimens to be used, thus expanding the therapeutic window and minimizing toxicity. Such an approach has the potential to allow the re-evaluation of agents that were previously determined to be too toxic. ${ }^{6}$

Cancer gene therapy has been at the forefront of keeping its readers up to date with these new advances. With original articles, reviews and editorials covering gene therapy, cellular therapy and immunotherapy from the most basic mechanistic studies to completed clinical trials, our goal has and will be to present the most important advances.

I want to take this opportunity to thank our founding editors $\mathrm{Dr}$ Sobol and Dr Scanlon for the vision and steadfast stewardship of the journal for the past 20 years. It is my sincere hope and intention to continue that legacy of excellence moving forward. It is my strong belief that the next 10 years will see an explosion of new mechanistic understanding and therapeutic translation of cancer gene and cellular therapies. CGT will remain at the forefront of bringing those findings to its readers as the premier journal in the field.

SK Libutti

E-mail: editor@cgtnature.com

\section{REFERENCES}

1 Siegel R, Naishadham D, Jemal A. Cancer statistics, 2013. CA Cancer J Clin 2013; 63: $11-30$.

2 Koldehoff M, Zakrzewski JL, Beelen DW, Elmaagacli AH. Additive antileukemia effects by GFI1B- and BCR-ABL-specific siRNA in advanced phase chronic myeloid leukemic cells. Cancer Gene Ther 2013; 20: 421-427.

3 Zhao J, Jin J, Zhang X, Shi M, Dai J, Wu M et al. Transfection of Smac sensitizes tumor cells to etoposide-induced apoptosis and eradicates established human hepatoma in vivo. Cancer Gene Ther 2006; 13: 420-427.

4 Kershaw MH, Westwood JA, Darcy PK. Gene-engineered T cells for cancer therapy. Nat Rev Cancer 2013; 13: 525-541.

5 Yuan Z, Syrkin G, Adem A, Geha R, Pastoriza J, Vrikshajanani C et al. Blockade of inhibitors of apoptosis (IAPs) in combination with tumor-targeted delivery of tumor necrosis factor- $\alpha$ leads to synergistic antitumor activity. Cancer Gene Ther 2013; 20: 46-56.

6 Citrin D, Camphausen K, Wood BJ, Quezado M, Denobile J, Pingpank JF et al. A pilot feasibility study of TNFerade ${ }^{\mathrm{TM}}$ biologic with capecitabine and radiation therapy followed by surgical resection for the treatment of rectal cancer. Oncology 2010; 79: $382-388$ 